\title{
Oil Red O and Hematoxylin: A Rapid Histologic Technic
}

\author{
KOGER W. CHOLEWIAK', LAWRENCE BUTCHER² AND NEIL M. KETTLEWELL \\ Department of Psychology, University of Michigan, Ann Arbor, Michigan, U.S.A.
}

(Received 8 March 1968)

\begin{abstract}
Cholewiak, R. W., L. Butcher and N. M. Kettlewell. Oil red $O$ and hematoxylin: a rapid histologic technic. Physiol. BeHAy. 3 (4) 585--586, 1968.- A rapid staining technique is described which considerably reduces the delay, relative to usual staining procedures, in the assessment of damage subsequent to brain lesions. Oil Red $\mathrm{O}$ and Harris' hematoxylin are used in the 15 min procedure. Coloration is excellent and distortion minimal.
\end{abstract}

Brain Histology Oil Red 0

IT IS NOT uncommon for those working in the area of brain research to spend considerable time in the preparation of appropriate histological data. Most conventional procedures involve one or more time-consuming steps, and thereby prohibit a rapid assessment of structural damage. When a sectioning technique employing paraffin is used, for example, the embedding process alone is usually an overnight procedure. Although there has been a gradual shift towards frozen sectioning in order to save time and effort, the subsequent staining procedure may itself take from several hours to days. Even if relatively short staining procedures are employed, for example the commonly used cresyl violet or Weil stains (several hours each), the time saved in sectioning is often offset by the disadvantage of staining either Nissl substance or myelin when the staining of both would be more desirable.

Unquestionably, there are situations in which the focus of research requires an elaborate histological scheme (i.e. the Marchi method for tracing degenerating nerve fibers). However, in verification of stereotaxic coordinates for electrode implantation, a reduction in the time for histology would be of considerable advantage.

A rapid technique is proposed using Oil Red $O$ stain with hematoxylin as a nucleic counterstain. French [2] and Proescher [6] describe Oil Red $O$ as a superior fat stain (therefore staining myelin) which has extremely good depth of color and yet leaves cellular structures intact. Humanson [4] and McManus and Mowry [5] describe its use with counterstains of luxol fast blue $B$ or hematoxylin which give excellent results but which are complicated by time-consuming or intricate schemes. The method to be described is relatively simple and takes approximately one-half hour from the removal of the perfused brain tissue to the completed slide. With adequate $\mathrm{CO}_{2}$ freezing, no problems have been encountered with the short perfusion. Shredding will result with sectioning of soft tissue. If desired, the tissue may be kept in $10 \%$ formalin overnight for deeper fixation.

\section{METHOD}

After the experimental manipulations, the animal (rat or rabbit), while still under anesthesia, is perfused with $10 \%$ formalin and the brain removed. Serial frozen sections are taken at $30-40 \mu$ in the region of interest and placed into a compartmentalized plastic tray filled with $20 \%$ ethyl alcohol. The sections are mounted on cleaned, numbered glass slides on which Fisher's albumin has been lightly spread. The mounting is done by floating the sections onto a coverslip from the tray and then on to the slides. They may be positioned on the slides with a fine sable brush.

Having allowed 5-10 min for the slides to dry, the staining procedure can begin. The dried slides are washed by dipping them one or two times in $70 \%$ alcohol, and they are then placed on an absorbant surface and covered with the Oil Red O solution $(1.5 \mathrm{~g}$ Oil Red $\mathrm{O}, 50.0 \mathrm{cc} 70 \%$ alcohol, $50.0 \mathrm{cc}$ acetone, mixed, filtered, and stored covered to prevent evaporation). The slides should be completely covered with the stain for approximately $5 \mathrm{~min}$. They are then washed in $70 \%$ alcohol followed by a distilled water wash. At this time the sections should be stained a bright orange-red with the fiber tracts particularly prominent. The slides are then counterstained by dropping on Harris' hematoxylin. This is prepared by heating and mixing a solution of $5.0 \mathrm{~g}$ hematoxylin dissolved in $50.0 \mathrm{cc} 95 \%$ alcohol with a solution of $100.0 \mathrm{~g}$ ammonium or potassium alum dissolved in $1000 \mathrm{cc}$ distilled water. The mixture is brought to a boil, removed from the heat, and $2.5 \mathrm{~g}$ mercuric oxide is added, after which it is reheated for about one min. It is ready for use when cooled and filtered (after Harris [3]). A prepared Harris' stain may be purchased from Scientific Glass Company, Harleco \#638X.

The counterstaining should not exceed 15-30 sec. The hematoxylin is washed from the slides with distilled water and is blued with a rapid wash in ammonia water (2-3 drops ammonium hydroxide in $1000 \mathrm{cc}$ distilled water). This is

'Now at the Department of Psychology, University of Virginia, Charlottesville, Virginia 22901.

${ }^{2}$ Now at Farmakologiska Institutionen, Göteborgs Universitet, Göteborg, Sweden. 
followed by a water wash. Differentiation is checked and the last step repeated if desired. Cover slips are then mounted on the slides with glycerin jelly $(10.0 \mathrm{~g}$ gelatin dissolved in $60.0 \mathrm{cc}$ water with $70.0 \mathrm{cc}$ glycerin and $1.0 \mathrm{cc}$ merthiolate. The jelly should be warmed for use).

The completed slides may be then cleaned with cold running water as long as care is taken not to displace the cover slip until the gelatin has hardened. For a more permanent preparation, the cover slip may be ringed with Permount.

The resulting sections will show fiber tracts bright orangered and cell nuclei blue. Lipoid degeneration products (lysed cell bodies) will have a deeper red cast [2].

An alternate technique which demonstrates only myelin sheaths involves placing the frozen sections in a warmed solution of the glycerin jelly, prepared as a 9:1 water: stock jelly mixture. After remaining in the solution for approximately $30 \mathrm{~min}$, the sections are mounted on slides and allowed to dry. The stain is then dropped on, as described above, and allowed to remain for $10-15 \mathrm{~min}$. The slides are then differentiated in $70 \%$ alcohol and washed in distilled water. Cover slips are then mounted as above.

Although the sectioning and mounting time will vary with the number of sections, the time necessary for staining with either of the above procedures should not exceed $15 \mathrm{~min}$. Much less distortion occurs due to shrinkage than with embedding techniques. The staining is deep and stable in the mounted sections [1], with no obvious fading after one year even with the gelatin cover slip mounting.

\section{REFERENCKS}

1. Conn, H. J. Biologic Stains (2nd ed.) Geneva N.Y.: Biotechnical Publications, 1929, p. 69.

2. French R. W. Notes on technic: fat stains. Stain Technol. 1: $78,1926$.

3. Harris, H. F. On the rapid conversion of haematoxylin into hamatein in staining reactions. J. appl. Microscop. 3: 777-780, 1900.
4. Humanson G. L. Animal Tissue Techmiques. San Francisco: Freeman \& Co., 1962.

5. McManus J. F. and R. W. Mowry. Staining Methods: Histologic and Histochemical. New York: Harper \& Row, 1965.

6. Proescher, F. Oil Red $O$ pyridin, a rapid fat stain. Stain Technol. 2: 60-61, 1927. 


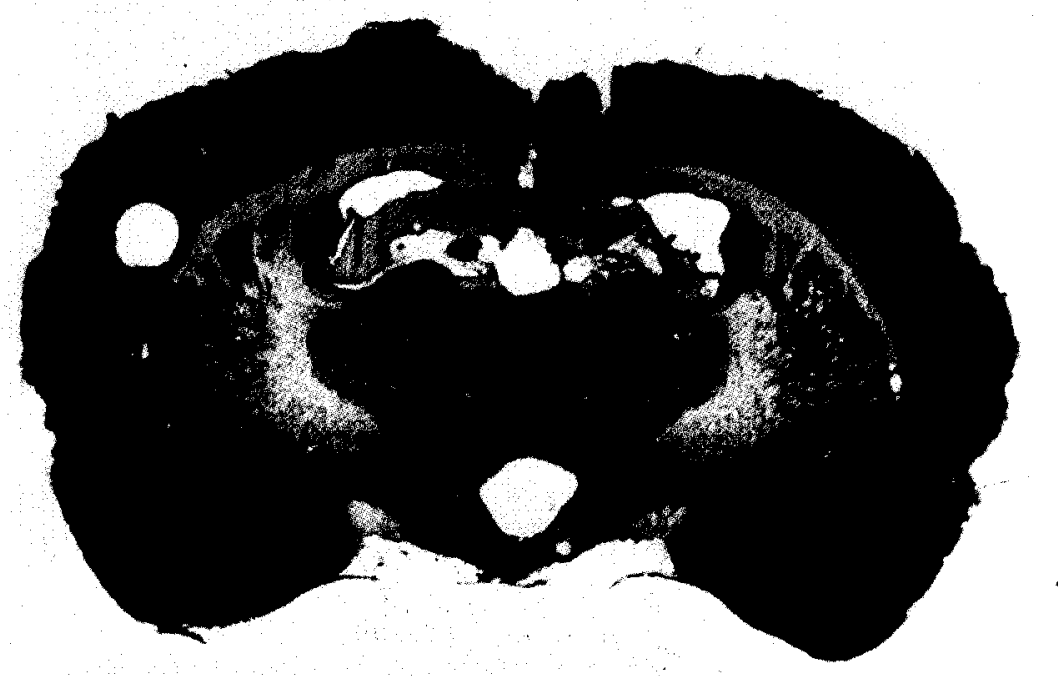

FIG. 1. Cross section of sample medial hypothalamic lesion

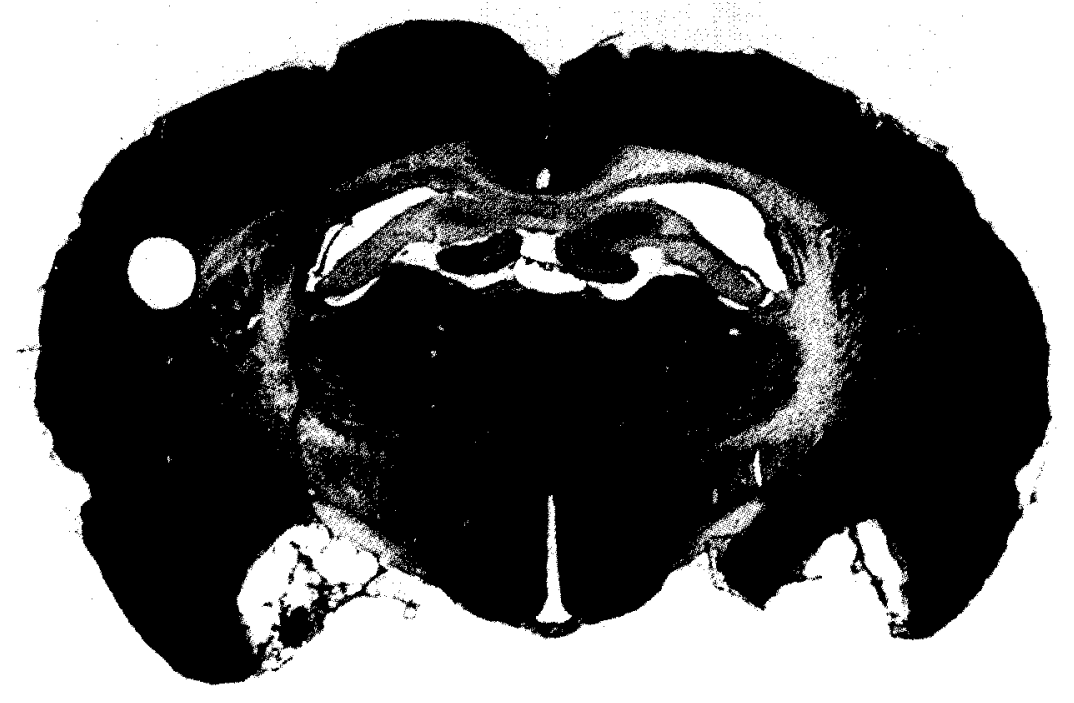

FIG. 2. Cross section of sample anterior amygdaloid complex lesion 


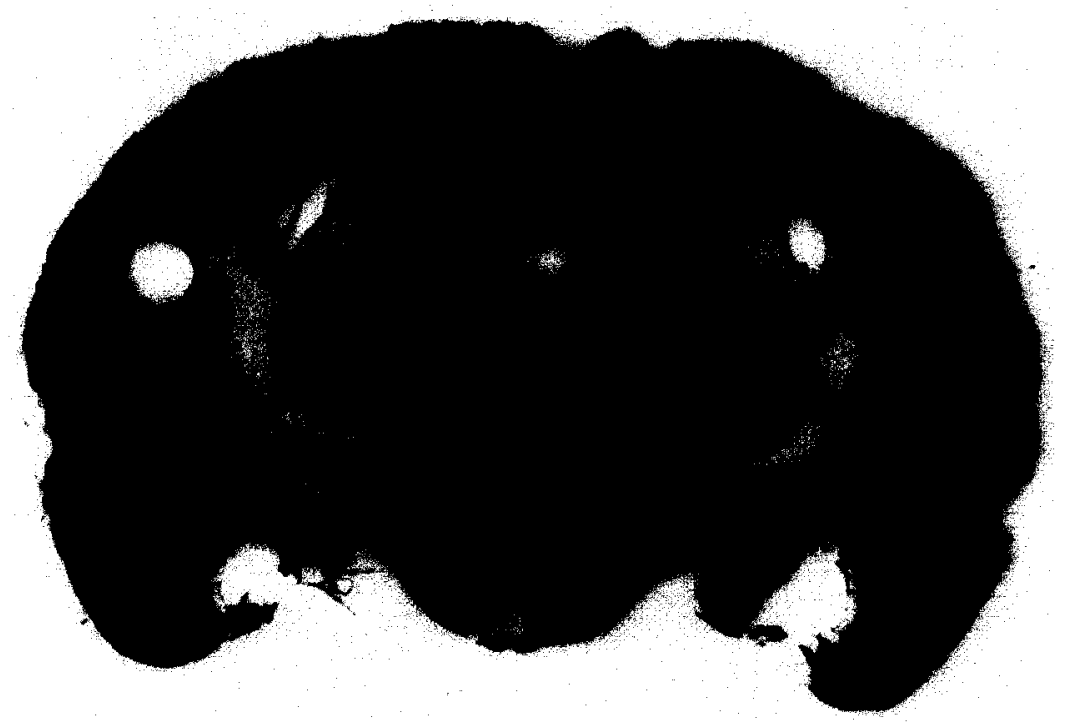

FIG. 3. Cross section of sample medial amygdaloid complex lesion 